\title{
AN EXTENSION TO THE BRUN-TITCHMARSH THEOREM
}

\author{
TSZ HO CHAN, STEPHEN KWOK-KWONG CHOI, AND KAI MAN TSANG
}

Abstract. The Siegel-Walfisz Theorem states that for any $B>0$, we have

$$
\sum_{\substack{p \leq x \\ p \equiv a(\bmod k)}} 1 \sim \frac{x}{\varphi(k) \log x}
$$

for $k \leq \log ^{B} x$ and $(k, a)=1$. This only gives an asymptotic formula for the number of primes over an arithmetic progression for quite small moduli $k$ compared to $x$. However, if we are only concerned about upper bound, we have the Brun-Titchmarsh theorem, namely, for any $1 \leq k<x$,

$$
\sum_{\substack{p \leq x \\ p \equiv a(\bmod k)}} 1 \ll \frac{x}{\varphi(k) \log (x / k)} .
$$

In this article, we prove an extension to the Brun-Titchmarsh theorem on the number of integers, with at most $s$ prime factors, in an arithmetic progression, namely,

$$
\sum_{\substack{y<n \leq x+y \\ n \equiv a(\bmod k) \\ \omega(n) \leq s}} 1 \ll \frac{x}{\varphi(k) \log (x / k)} \sum_{\ell=0}^{s-1} \frac{(\log \log (x / k)+K)^{\ell}}{\ell !}
$$

for any $x, y>0, s \geq 1$ and $1 \leq k<x$.

In particular, for $s \leq \log \log (x / k)$, we have

$$
\sum_{\substack{y<n \leq x+y \\ n=a(\bmod k) \\ \omega(n) \leq s}} 1 \ll \frac{x}{\varphi(k) \log (x / k)} \frac{(\log \log (x / k)+K)^{s-1}}{(s-1) !} \sqrt{\log \log (x / k)+K}
$$

and for any $\varepsilon \in(0,1)$ and $s \leq(1-\varepsilon) \log \log (x / k)$, we have

$$
\sum_{\substack{y<n \leq x+y \\ n \equiv a(\bmod k) \\ \omega(n) \leq s}} 1 \ll \frac{\varepsilon^{-1} x}{\varphi(k) \log (x / k)} \frac{(\log \log (x / k)+K)^{s-1}}{(s-1) !} .
$$

Date: December 14, 2009.

Research of K.K. Choi is supported by NSERC of Canada.

Research of K.M. Tsang is fully supported by RGC grant HKU 7042/04P of the Hong Kong SAR, China. 


\section{INTRODUCTION}

Throughout this paper, $p, p_{1}, p_{2}, \ldots$ etc. denote prime numbers.

The celebrated Prime Number Theorem, conjectured by Gauss and proved by Hadamard and de la Vallée Poussin, asserts that

$$
\sum_{p \leq x} 1=(1+o(1)) \frac{x}{\log x}
$$

where the summation is over all primes $p \leq x$. One can also consider the distribution of primes in arithmetic progressions. For $(a, k)=1$, put

$$
\sum_{\substack{p \leq x \\ p \equiv a(\bmod k)}} 1=\frac{\operatorname{Li}(x)}{\varphi(k)}+E(x ; k, a),
$$

where $\operatorname{Li}(x):=\int_{0}^{x} \frac{1}{\log t} d t$ and $\varphi(n)$ is the Euler function. The Siegel-Walfisz theorem states that for arbitrary constants $A, B>0$

$$
\max _{(a, k)=1}|E(x ; k, a)| \ll \frac{x}{k}(\log x)^{-A}
$$

uniformly for $k \leq(\log x)^{B}$ and this only gives an asymptotic estimate (1) for quite small moduli $k$ compared to $x$. Furthermore, if we assume the generalized Riemann hypothesis, we can prove that (2) holds for $k \leq x^{1 / 2}(\log x)^{-A-2}$. The celebrated Bombieri-Vinogradov theorem has the same strength as the generalized Riemann hypothesis on average, and therefore enables us to deal with various problems. It asserts that for every $A>0$ there exists a constant $B=B(A)>0$ such that

$$
\sum_{k \leq x^{1 / 2}(\log x)^{-B}} \max _{(a, k)=1}|E(x ; k, a)| \ll \frac{x}{(\log x)^{A}} .
$$

See [1], for instance. Although asymptotic estimate (1) can only be proved so far for quite small moduli $k$, if we are only concerned about upper bound inequality for (1), we have the Brun-Titchmarsh theorem (e.g see [2] ), namely, for any $1 \leq k<x$,

$$
\sum_{\substack{p \leq x \\ p \equiv a(\bmod k)}} 1 \ll \frac{x}{k \log (x / k)} .
$$

In this article, we consider an extension of the Brun-Titchmarsh theorem and study the number of integers in short intervals with exactly $s$ prime factors (counted with multiplicity) that lie in the arithmetic progression $a(\bmod k)$. Let $\Omega(n)$ denote the number of prime factors of $n$ (counted with multiplicity) and $\omega(n)$ denote the 
number of distinct prime factors of $n$. Landau [5] showed that for fixed $s$ the asymptotic formula

$$
\sum_{\substack{n \leq x \\ \omega(n)=s}} 1=(1+o(1)) \frac{x}{\log x} \frac{(\log \log x)^{s-1}}{(s-1) !}
$$

holds. Sathe [8] and Selberg [9] proved a more precise quantitative estimate. Let

$$
F(z):=\frac{1}{\Gamma(z+1)} \prod_{p}\left(1+\frac{z}{p-1}\right)\left(1-\frac{1}{p}\right)^{z}
$$

where the product is taken over all primes $p$. Then

$$
\sum_{\substack{n \leq x \\ \omega(n)=s}} 1=F\left(\frac{s}{\log \log x}\right) \frac{x}{\log x} \frac{(\log \log x)^{s-1}}{(s-1) !}\left(1+O\left(\frac{1}{\log \log x}\right)\right)
$$

holds uniformly for $x \geq 3$ and $1 \leq s \leq C \log \log x$ for any given fixed $C>0$. For more discussions, see [4] and [6].

Upper bounds for the above sum have been obtained for much wider ranges. A classical example is the Hardy-Ramanujan inequality [3]

$$
\sum_{\substack{n \leq x \\ \omega(n)=s}} 1 \leq c_{1} \frac{x}{\log x} \frac{\left(\log \log x+c_{2}\right)^{s-1}}{(s-1) !}
$$

which is valid, with suitable constants $c_{1}$ and $c_{2}$, for all $x \geq 3$ and $s \geq 1$. In [12], Warlimont and Wolke extended the Hardy-Ramanujan inequality by estimating the number of such integers in an interval $(y, x+y]$, namely,

$$
\sum_{\substack{y<n \leq y+x \\ \omega(n)=s}} \mu^{2}(n) \leq \frac{c_{1}}{\epsilon} \frac{x}{\log x} \frac{\left(\log \log x+c_{2}\right)^{s-1}}{(s-1) !}
$$

for any $y \geq 0$ and $0<\epsilon<1 / 2$, provided that for sufficiently large $x$,

$$
1 \leq s \leq(1-\epsilon) \frac{\log \log x}{\log \log \log x} .
$$

It was mentioned in [12] that, a larger upper bound than (4) is needed if the range of $s$ is extended to $1 \leq s \ll \log \log x$. A further remark on this will be discussed later. Recently, Tudesq in [11] showed that the inequality (4) still holds when the squarefree condition is removed but for a larger upper bound. He proved that for $2 \leq x \leq y$ and $s \geq 1$,

$$
\sum_{\substack{y<n \leq y+x \\ \omega(n)=s}} 1 \ll \frac{x}{\log x} \frac{\left(\log \log x+c_{2}\right)_{s-1}}{(s-1) !} .
$$


Here $(t)_{r}:=t(t+1) \cdots(t+r-1)$. It can be easily shown that

$$
(t)_{s-1}=t^{s-1}\left(1+\frac{1}{t}\right) \cdots\left(1+\frac{s-2}{t}\right) \leq t^{s-1} \exp \left\{\frac{(s-2)(s-1)}{2 t}\right\}
$$

and hence in the range $1 \leq s \ll \sqrt{\log \log x}$, Tudesq's result recovers Warlimont and Wolke's result (4).

By extending Selberg's idea in [9], Spiro proved in [10], among other results, that if we fix $B^{\prime}$ with $0<B^{\prime}<B(B=2$ for $\eta=\Omega$ and $B$ is arbitrary for $\eta=\omega)$, select $u>0$, and let $a, s, k$ be integers with $(a, k)=1$,

$$
1 \leq s \leq B^{\prime} \log \log x
$$

and

$$
1 \leq k<\exp (\sqrt{\log x}), \quad \prod_{p \mid k} p \leq(\log x)^{u},
$$

then for $\eta(n)=\omega(n)$ or $\Omega(n)$, we have

$$
\sum_{\substack{n \leq x \\ n \equiv a(\bmod k) \\ \eta(n)=s}} 1=(1+o(1)) G(y) \frac{x}{\varphi(k) \log x} \cdot \frac{(\log \log x)^{s-1}}{(s-1) !}\left(\frac{\varphi(k)}{k}\right)^{y}
$$

uniformly in $a, s$ and $k$, where $y=\frac{s-1}{\log \log x}, \chi_{0}$ is the principal character modulo $k$, $G(z):=\frac{g\left(1, z, \chi_{0} ; \eta\right)}{\Gamma(1+z)}$ and

$$
g(w, z, \chi ; \eta):=\prod_{p}\left(\sum_{m=0}^{\infty} \chi\left(p^{m}\right) z^{\eta\left(p^{m}\right)} p^{-m w}\right)\left(1-\chi(p) p^{-w}\right)^{z}
$$

wherever the product converges.

For larger values of $k$ the situation is much less satisfactory and no simple asymptotic formulae have been obtained so far. The purpose of this paper is to obtain an upper bound estimate for the number of positive integers in the interval $(y, x+y]$ which have exactly $s$ prime factors and lie in the arithmetic progression $a(\bmod k)$ for large range of $k$. In [7], Orazov showed that for fixed $s \geq 1$,

$$
\sum_{\substack{y<n \leq x+y \\ n \equiv a(\bmod k) \\ \omega(n)=s}} \mu^{2}(n) \ll_{s} \frac{\tau(k)^{s-1} x(\log \log x)^{s-1}}{\varphi(k) \log x}
$$

provided $k \leq x^{1 /(2 s)}$, where $\tau(k)$ is the divisor function of $k$.

Our main Theorem is the following. 
Theorem 1. Let $x, y>0$ and $s \geq 1$ and let $a, k$ be coprime positive integers with $1 \leq k<x$. We have

$$
\sum_{\substack{y<n \leq x+y \\ n=a \text { (mod } \\ \omega(n) \leq s}} 1 \leq \frac{K x}{\varphi(k) \log (x / k)} \sum_{\ell=0}^{s-1} \frac{(\log \log (x / k)+K)^{\ell}}{\ell !} .
$$

In particular, for $s \leq \log \log (x / k)$, the right hand side of (6) is

$$
\leq \frac{K x}{\varphi(k) \log (x / k)} \frac{(\log \log (x / k)+K)^{s-1}}{(s-1) !} \sqrt{\log \log (x / k)+K} ;
$$

and for any $\varepsilon \in(0,1)$ and $s \leq(1-\varepsilon) \log \log (x / k)$, the right hand side is

$$
\leq \frac{K \varepsilon^{-1} x}{\varphi(k) \log (x / k)} \frac{(\log \log (x / k)+K)^{s-1}}{(s-1) !} .
$$

For any $L \geq 1$, it is not difficult to show that

$$
\sum_{\ell=0}^{L} \frac{L^{\ell}}{\ell !} \asymp \sum_{|\ell-L| \leq \sqrt{L}} \frac{L^{\ell}}{\ell !} \asymp \sqrt{L} \frac{L^{L}}{L !} \asymp e^{L} .
$$

Hence, when $s \geq \log \log (x / k)+K$, the sum in the right hand side of (6) is $\gg$ $\log (x / k)$, yielding a trivial bound for the sum in the left hand side. Thus the main interest of our result concerns the range $1 \leq s \leq \log \log (x / k)$.

Clearly Theorem 1 includes the following.

Theorem 2. Let $x, y>0$ and let $a, k$ be coprime positive integers with $1 \leq k<x$. For $\eta(n)=\omega(n)$ or $\Omega(n)$, we have

$$
\sum_{\substack{y<n \leq x+y \\ n \equiv a(\bmod k) \\ \eta(n)=s}} 1 \leq \frac{K x}{\varphi(k) \log (x / k)} \sum_{\ell=0}^{s-1} \frac{(\log \log (x / k)+K)^{\ell}}{\ell !} .
$$

In particular, for $s \leq \log \log (x / k)$, we have

$$
\sum_{\substack{y<n \leq x+y \\ n=a \\ n(n)=s}} 1 \leq \frac{K x}{\varphi(k) \log (x / k)} \frac{(\log \log (x / k)+K)^{s-1}}{(s-1) !} \sqrt{\log \log (x / k)+K} ;
$$

and for any $\varepsilon \in(0,1)$ and $s \leq(1-\varepsilon) \log \log (x / k)$, we have

$$
\sum_{\substack{y<n \leq x+y \\ n=a(\bmod k) \\ \eta(n)=s}} 1 \leq \frac{K \varepsilon^{-1} x}{\varphi(k) \log (x / k)} \frac{(\log \log (x / k)+K)^{s-1}}{(s-1) !} .
$$


Our Theorem 2 improves Warlimont and Wolke's, Tudesq's and Orazov's results.

We remark that in our range of $1 \leq s \leq(1-\varepsilon) \log \log (x / k),(5)$ shows that our upper bound in Theorem 2 is of the correct order of magnitude, at least when $x$ is a sufficiently large function of $y$.

It is very tempting to extend (7) in our Theorem 2 to the more natural range $1 \leq s \leq(1+\varepsilon) \log \log (x / k)$. However, similar to the comment of Warlimont and Wolke in [12], this can't be done. We prove this in the following proposition.

Proposition 3. For any $A$ and $\epsilon>0$, there exist constants $c_{0}(A, \epsilon)$ and $c_{1}(A, \epsilon)$ with the following property: Let $x \geq c_{0}, 1 \leq k \leq \exp \left(\sqrt{\log x}-\frac{1}{2}\right), \prod_{p \mid k} p \leq \log x$ and

(8) $\log \log (x / k)+2 \sqrt{\log \log (x / k) \log (2 k / \varphi(k))}$

$$
+c_{1} \sqrt{\log \log (x / k)} \leq s \leq(2-\epsilon) \log \log (x / k) .
$$

Then for any positive integer a coprime with $k$, there exists $y>0$ such that

$$
\sum_{\substack{y<n \leq y+x \\ n=a(\bmod k) \\ \Omega(n)=s}} 1 \geq A \frac{x}{\varphi(k) \log (x / k)} \frac{(\log \log (x / k))^{s-1}}{(s-1) !} .
$$

Proof. Since $1 \leq k \leq \exp \left(\sqrt{\log x}-\frac{1}{2}\right)$, we have $1 \leq k \leq \exp (\sqrt{\log (x / k)})$. Then we apply Spiro's result in (5) with $Y=\exp \left((\log (x / k))^{1+\delta}\right)$ in place of $x$, where $\delta>0$ is to be determined later. We note that (c.f. Lemma 3 in [10]) for $s$ satisfying condition (8), we have $(s-1) / \log \log Y<(2-\epsilon) /(1+\delta)<2-\epsilon$ and

$$
1 \ll G\left(\frac{s-1}{\log \log Y}\right)=\Gamma\left(1+\frac{s-1}{\log \log Y}\right)^{-1} g\left(1, \frac{s-1}{\log \log Y}, \chi_{0} ; \Omega\right) \ll 1
$$

Hence, by (5)

$$
\sum_{\substack{n \leq Y \\ n=a(\bmod k) \\ \Omega(n)=s}} 1 \geq c_{3} \frac{Y}{\varphi(k) \log Y} \frac{(\log \log Y)^{s-1}}{(s-1) !}\left(\frac{\varphi(k)}{k}\right)^{(s-1) / \log \log Y}
$$

for some constant $c_{3}(\epsilon)>0$. 
Partition the interval $(0, Y]$ into $\leq[Y / x]+1$ subintervals of the form $(0, x],(x, 2 x]$, $(2 x, 3 x], \ldots$. Then there exists $y=j x>0$ for some $j$ such that

$$
\begin{aligned}
\sum_{\substack{y<n \leq y+x \\
n=a(\bmod k) \\
\Omega(n)=s}} 1 & \geq \frac{1}{[Y / x]+1} \sum_{\substack{n \leq Y \\
n \equiv a(\bmod k) \\
\Omega(n)=s}} 1 \\
& \geq \frac{c_{3}}{2} \frac{x}{\varphi(k) \log Y} \frac{(\log \log Y)^{s-1}}{(s-1) !}\left(\frac{\varphi(k)}{k}\right)^{(s-1) / \log \log Y} .
\end{aligned}
$$

Comparing the right hand side with the desired lower bound in our proposition, we see that our proposition would follow if

$$
s-1 \geq\left(\delta \log \log (x / k)+\log \left(2 A / c_{3}\right)\right)\left(\log (1+\delta)-\frac{1}{\log \log Y} \log (k / \varphi(k))\right)^{-1}
$$

holds. Using now the inequalities $\log (1+\delta) \geq \delta-\frac{1}{2} \delta^{2}$ and $\log \log Y>\log \log (x / k)$, the right hand side above is

$$
\leq\left(\delta \log \log (x / k)+\log \left(2 A / c_{3}\right)\right)\left(\delta-\frac{1}{2} \delta^{2}-\log (k / \varphi(k)) / \log \log (x / k)\right)^{-1} .
$$

By taking $\delta$ satisfying

$$
\frac{1}{2} \delta^{2}=\log (2 k / \varphi(k)) / \log \log (x / k),
$$

we find that the last expression is

$$
\leq \log \log (x / k)+2 \sqrt{\log \log (x / k) \log (2 k / \varphi(k))}+2 \log \left(2 A / c_{3}\right) \sqrt{\log \log (x / k)} .
$$

This proves our proposition.

Our argument in Proposition 3 does not work if the interval $(y, y+x]$ is replaced by the interval $(1, x]$. In fact, the counterexample occurs when $y$ is quite large, namely, $\log y=(\log (x / k))^{1+\delta}$.

The main idea of proof of our Theorem 1 is the classification of positive integers $n=\left(p_{1}^{\alpha_{1}} \cdots p_{j-1}^{\alpha_{j-1}}\right)\left(p_{j}^{\alpha_{j}} \cdots p_{\ell}^{\alpha_{\ell}}\right)=r w$, say where $j$ is determined by

$$
p_{1}^{\alpha_{1}} p_{2}^{\alpha_{2}} \cdots p_{j-1}^{\alpha_{j-1}+1}<\frac{x}{k} \leq p_{1}^{\alpha_{1}} p_{2}^{\alpha_{2}} \cdots p_{j}^{\alpha_{j}+1} .
$$

Then $\sum_{n}=\sum_{r} \sum_{w}$. In each $w$, either $p_{j}$ is large or $p_{j}^{\alpha_{j}}$ is a large prime power. In the former case, $\sum_{w}$ can be estimated by sieve method. When $p_{j}^{\alpha_{j}}$ is a large prime power, there are at most two possibilities for the exponent $\alpha_{j}$ and a trivial estimate for the $\sum_{w}$ then suffices. The sum $\sum_{r}$ can be estimated by Hardy-Ramanujan type argument in [3]. This yields the $(j-1)$ ! in the denominator in Lemma 8. 


\section{LEMMAS}

We need the following results to prove our Theorem 1. Throughout, we let $K$ denote a positive absolute constant which need not have the same value at each occurrence but $K$ is effectively computable.

Lemma 4. For $2 \leq y<z<X$, we have

$$
\begin{aligned}
\sum_{y<p \leq z} \frac{1}{p \log (X / p)}=\frac{1}{\log X}\left(\log \left(\frac{\log z}{\log (X / z)}\right)-\log \left(\frac{\log y}{\log (X / y)}\right)\right) & \\
& +O\left(\frac{1}{\log z \log (X / z)}+\frac{1}{\log y \log (X / y)}\right) .
\end{aligned}
$$

In particular, for $2 \leq z \leq \sqrt{X}$, we have

$$
\sum_{p \leq z} \frac{1}{p \log (X / p)} \leq \frac{\log \log z+K}{\log X}
$$

Proof. This lemma can be proved by the partial summation that

$$
\sum_{y<p \leq z} \frac{1}{p \log (X / p)}=\left.\frac{1}{\log (X / t)} \sum_{p \leq t} \frac{1}{p}\right|_{y} ^{z}-\int_{y}^{z}\left(\sum_{p \leq t} \frac{1}{p}\right) \frac{d t}{t \log ^{2}(X / t)}
$$

and the well-known estimate

$$
\sum_{p \leq t} \frac{1}{p}=\log \log t+c+O\left(\frac{1}{\log t}\right)
$$

for $t \geq 2$ and some constant $c$.

For any $w>1$, we let

$$
P(w):=\prod_{p<w} p
$$

where the product is over all primes less than $w$.

Lemma 5. For any $X \geq 1, Y>0$ and any integers $a$ and $k>0$ such that $(a, k)=1$, we have

$$
\sum_{\substack{Y<n \leq X+Y \\(n, P(w))=1 \\ n \equiv a(\bmod k)}} 1 \leq K\left(\frac{X}{\varphi(k) \log w}+\frac{k w^{2}}{\varphi(k) \log ^{2} w}\right) .
$$

Proof. This is Theorem 3.6 of [2] or Theorem 3.8 of [6]. 
Lemma 6. Let $X, Y>0$ and let $a, k$ be coprime positive integers with $1 \leq k<X$. We have

$$
\sum_{\substack{Y<p^{\alpha} \leq X+Y \\ p^{\alpha} \equiv a(\bmod k)}} 1 \leq \frac{K X}{\varphi(k) \log (X / k)} .
$$

Here the summation on the left hand side is over all prime powers $p^{\alpha}$.

Proof. For $k>X / 2$, the result is trivial. We may henceforth assume $k \leq X / 2$. If $p>\sqrt{\frac{X}{k}}$, then the sieve bound Lemma 5 yields our result as before.

For any prime $p$, let $1 \leq \beta_{1}<\beta_{2}<\cdots$ be such that

$$
Y<p^{\beta_{i}} \leq X+Y, p^{\beta_{i}} \equiv a(\bmod k), i=1,2, \ldots
$$

Then for $j>i, p^{\beta_{j}}-p^{\beta_{i}}$ is a multiple of $k$ and so is $\left(p^{\beta_{j}-\beta_{i}}-1\right)$ since $(p, k)=$ $(a, k)=1$. Also,

$$
X>p^{\beta_{j}}-p^{\beta_{i}}=p^{\beta_{i}}\left(p^{\beta_{j}-\beta_{i}}-1\right)>0 .
$$

Hence

$$
p^{\beta_{i}}<X / k
$$

In other words, all but one of the prime powers $p^{\alpha}$ in the sum of the lemma satisfy

$$
p^{\alpha}<X / k \text {. }
$$

In particular, for each $p$ there are $\ll \log (X / k)+1$ powers of $p$ satisfying (10). Thus

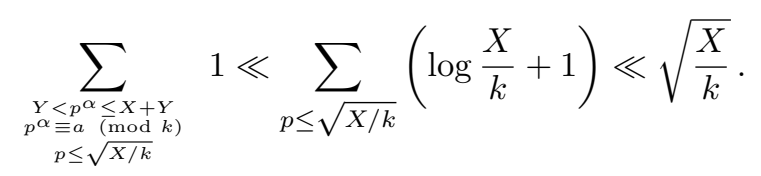

This completes the proof of our lemma.

For any positive integer $m=p_{1}^{\alpha_{1}} p_{2}^{\alpha_{2}} \cdots p_{j}^{\alpha_{j}}$, where $p_{1}<p_{2}<\cdots<p_{j}$ are primes and $\alpha_{1}, \alpha_{2}, \ldots, \alpha_{j} \geq 1$ we let

$$
u(m):=p_{1}^{\alpha_{1}} p_{2}^{\alpha_{2}} \cdots p_{j-1}^{\alpha_{j-1}} p_{j}^{\alpha_{j}+1},
$$

and

$$
v(m):=p_{1}^{\alpha_{1}} p_{2}^{\alpha_{2}} \cdots p_{j-1}^{\alpha_{j-1}} p_{j}^{2} .
$$

Define $u(1)=v(1)=1$. 
Lemma 7. Let $\xi \geq 2$. Define $P_{0}(\xi):=\frac{1}{\log \xi}$. For $h \geq 1$, we have

$$
P_{h}(\xi):=\sum_{\substack{m=p_{1}^{\alpha_{1} \ldots} p_{h}^{\alpha_{h}} \\ u(m) \leq \xi}}(m \log (\xi / m))^{-1} \leq \frac{1}{h ! \log \xi}(\log \log \xi+K)^{h}
$$

and

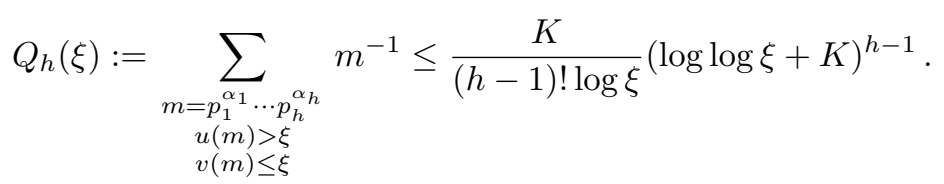

Proof. First,

$$
\begin{aligned}
P_{1}(\xi)= & \sum_{\substack{p^{\alpha} s . t . \\
p^{\alpha+1} \leq \xi}}\left(p^{\alpha} \log \left(\xi p^{-\alpha}\right)\right)^{-1} \\
= & \sum_{p \leq \sqrt{\xi}}\left(p \log \left(\xi p^{-1}\right)\right)^{-1}+\sum_{p \leq \xi^{1 / 3}} \sum_{\alpha=2}^{\left[\frac{\log \xi / p}{\log p}\right]}\left(p^{\alpha} \log \left(\xi p^{-\alpha}\right)\right)^{-1} \\
= & \frac{1}{\log \xi}\left(\log \left(\frac{\log \xi^{1 / 2}}{\log \xi^{1 / 2}}\right)-\log \left(\frac{\log 2}{\log (\xi / 2)}\right)\right)+O\left(\frac{1}{\log \xi}\right) \\
& \quad+O\left(\sum_{p \leq \xi^{1 / 3}} \sum_{\alpha=2}^{\left[\frac{\log \xi / p}{\log p}\right]} \frac{\alpha+1}{\log \xi} p^{-\alpha}\right) \\
\leq & \frac{1}{\log \xi}(\log \log \xi+K)+O\left(\sum_{p \leq \xi^{1 / 3}} \frac{1}{p^{2} \log \xi}\right) \\
\leq & \frac{1}{\log \xi}(\log \log \xi+K)
\end{aligned}
$$

by Lemma 4 . We now use mathematical induction on $h \geq 1$. For each prime power $p^{\alpha}, \alpha \geq 1$, let

$$
\mathcal{L}\left(p^{a}\right):=\left\{m p^{\alpha}:(p, m)=1, \omega(m)=h, u\left(m p^{\alpha}\right) \leq \xi\right\} .
$$

For each $n$ counted in the sum $P_{h+1}(\xi)$, we have $n=p_{1}^{\alpha_{1}} p_{2}^{\alpha_{2}} \cdots p_{h+1}^{\alpha_{h+1}}, u(n) \leq \xi$. Hence $n$ belongs to $\mathcal{L}\left(p_{i}^{\alpha_{i}}\right)$ for $i=1,2, \ldots, h+1$ and

$$
(h+1) P_{h+1}(\xi)=\sum_{p^{\alpha}} \sum_{n \in \mathcal{L}\left(p^{\alpha}\right)}\left(n \log \frac{\xi}{n}\right)^{-1}
$$


For $n=m p^{\alpha} \in \mathcal{L}\left(p^{\alpha}\right)$, the condition $u\left(m p^{\alpha}\right) \leq \xi$ implies $u(m) \leq \xi / p^{\alpha}$. Also $\mathcal{L}\left(p^{\alpha}\right)$ is an empty set unless $p^{\alpha+1} \leq \xi$. Thus, the last double sum is

$$
\begin{aligned}
& \leq \sum_{\substack{p^{\alpha} \text { s.t. } \\
p^{\alpha+1} \leq \xi}} p^{-\alpha} P_{h}\left(\xi / p^{\alpha}\right) \\
& \leq \frac{1}{h !} \sum_{p^{\alpha+1} \leq \xi} p^{-\alpha} \frac{\left(\log \log \left(\xi / p^{\alpha}\right)+K\right)^{h}}{\log \left(\xi / p^{\alpha}\right)},
\end{aligned}
$$

by induction hypothesis on $P_{h}\left(\xi / p^{\alpha}\right)$. Then by $P_{1}(\xi)$, we find that

$$
\begin{aligned}
P_{h+1}(\xi) & \leq \frac{1}{(h+1) !}(\log \log \xi+K)^{h} \sum_{p^{\alpha+1} \leq \xi}\left(p^{\alpha} \log \left(\xi / p^{\alpha}\right)\right)^{-1} \\
& \leq \frac{1}{(h+1) ! \log \xi}(\log \log \xi+K)^{h+1},
\end{aligned}
$$

as desired.

Next we prove the bound for $Q_{j}(\xi)$.

$$
\begin{aligned}
Q_{1}(\xi) & =\sum_{\substack{p^{\alpha} s . t . \\
p^{\alpha+1}>\xi \\
p^{2} \leq \xi}} p^{-\alpha}=\sum_{p \leq \sqrt{\xi}} \sum_{\alpha+1>\log \xi} p^{-\alpha} \log p \\
& \leq \sum_{p \leq \sqrt{\xi}} \frac{p^{2} \xi^{-1}}{p-1}=\xi^{-1} \sum_{p \leq \sqrt{\xi}}\left(p+1+\frac{1}{p-1}\right) \leq \frac{K}{\log \xi} .
\end{aligned}
$$

For $h \geq 2$, using the above bound for $Q_{1}(\xi)$, we have

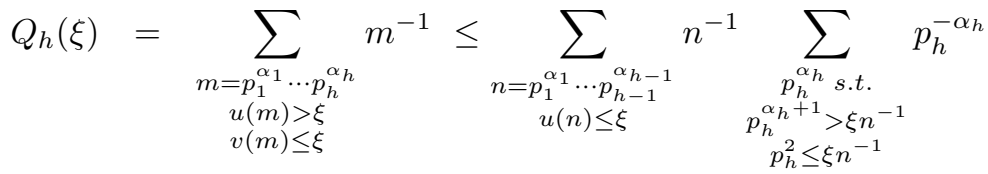

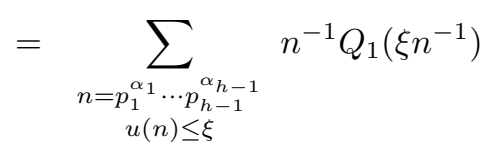

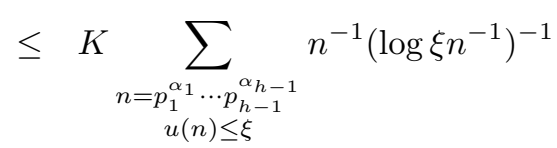

$$
\begin{aligned}
& =K P_{h-1}(\xi) \leq \frac{K}{(h-1) ! \log \xi}(\log \log \xi+K)^{h-1},
\end{aligned}
$$

as desired.

For each integer $n>1$, let $n=p_{1}^{\alpha_{1}} \cdots p_{t}^{\alpha_{t}}$ denote its canonical factorization in which $p_{1}<p_{2}<\cdots<p_{t}$. For any $\xi>1$, define the following sets:

$$
H_{1}=H_{1}(\xi):=\left\{n: \omega(n) \geq 1, p_{1}^{\alpha_{1}+1}>\xi\right\}
$$




$$
\begin{aligned}
& H_{2}=H_{2}(\xi):=\left\{n: \omega(n) \geq 2, p_{1}^{\alpha_{1}+1} \leq \xi, p_{1}^{\alpha_{1}} p_{2}^{\alpha_{2}+1}>\xi\right\} \\
& H_{3}=H_{3}(\xi):=\left\{n: \omega(n) \geq 3, p_{1}^{\alpha_{1}} p_{2}^{\alpha_{2}+1} \leq \xi, p_{1}^{\alpha_{1}} p_{2}^{\alpha_{2}} p_{3}^{\alpha_{3}+1}>\xi\right\}
\end{aligned}
$$

and in general, for $j \geq 1$,

$$
H_{j}=H_{j}(\xi)=\left\{n: \omega(n) \geq j, p_{1}^{\alpha_{1}} \cdots p_{j-2}^{\alpha_{j-2}} p_{j-1}^{\alpha_{j-1}+1} \leq \xi, p_{1}^{\alpha_{1}} \cdots p_{j-1}^{\alpha_{j-1}} p_{j}^{\alpha_{j}+1}>\xi\right\}
$$

Lemma 8. Let $x, y>0$. For any coprime positive integers $a, k$ with $k \leq \frac{x}{2}$ and $\xi=x / k$, we have

$$
\sum_{\substack{y<n \leq x+y \\ n \equiv a(\bmod k) \\ n \in H_{j}}} 1 \leq \frac{K x}{\varphi(k) \log (x / k)} \frac{(\log \log (x / k)+K)^{j-1}}{(j-1) !} .
$$

Proof. We split the set $H_{j}$ into

$$
\begin{aligned}
& H_{j}=\left\{n: p_{1}^{\alpha_{1}} \cdots p_{j-2}^{\alpha_{j-2}} p_{j-1}^{\alpha_{j-1}+1} \leq \xi, p_{1}^{\alpha_{1}} \cdots p_{j-1}^{\alpha_{j-1}} p_{j}^{2}>\xi\right\} \\
& \cup\left\{n: p_{1}^{\alpha_{1}} \cdots p_{j-1}^{\alpha_{j-1}} p_{j}^{2} \leq \xi, p_{1}^{\alpha_{1}} \cdots p_{j-1}^{\alpha_{j-1}} p_{j}^{\alpha_{j}+1}>\xi\right\} \\
&=: \quad H_{j}^{(1)} \cup H_{j}^{(2)}
\end{aligned}
$$

say. For each $n \in H_{j}$, write $n=r w$ where

$$
r=p_{1}^{\alpha_{1}} \cdots p_{j-1}^{\alpha_{j-1}}, w=\frac{n}{r}
$$

Note $w>1$, and $r=1$ if $j=1$. Furthermore, for $n \equiv a(\bmod k)$, we have $(r, k)=1$ since $(a, k)=1$. If $n \in H_{j}^{(1)}$, then the smallest prime factor in $w$ is $p_{j}$, which is bigger than $\sqrt{\xi / r}$. Hence

$$
\begin{aligned}
\sum_{\substack{y<n \leq x+y \\
n \equiv a(\bmod k) \\
n \in H_{j}^{(1)}}} 1 \leq \sum_{r} \sum_{\substack{y r^{-1}<w \leq(x+y) r^{-1} \\
w \equiv \bar{r} a(\bmod k) \\
\left(w, P\left(\sqrt{\frac{x}{k r}}\right)\right)=1}} 1 \\
\leq K \sum_{r} \frac{x}{r \varphi(k) \log \frac{x}{k r}}
\end{aligned}
$$

by applying the sieve bound in Lemma 5 . 
The summation $\sum_{r}$ is exactly that in $P_{j-1}(x / k)$. Thus,

$$
\begin{aligned}
\sum_{\substack{y<n \leq x+y \\
n \equiv a(\bmod k) \\
n \in H_{j}^{(1)}}} 1 & \leq \frac{K x}{\varphi(k)} \sum_{r}\left(r \log \frac{x}{k r}\right)^{-1}=\frac{K x}{\varphi(k)} P_{j-1}(x / k) \\
& \leq \frac{K x}{\varphi(k) \log (x / k)} \frac{(\log \log (x / k)+K)^{j-1}}{(j-1) !}
\end{aligned}
$$

by Lemma 7. Before we move on, we take note of the following simple fact: if $y<n<n^{\prime} \leq x+y$ and $n \equiv a \equiv n^{\prime}(\bmod k)$, then $n^{\prime}-n$ is divisible by $k$ as well as $\operatorname{gcd}\left(n, n^{\prime}\right)$. Furthermore since $(n, k)=1=\left(n^{\prime}, k\right)$, we can conclude that $n^{\prime}-n$ is a positive multiple of $k\left(n, n^{\prime}\right)$. In particular,

$$
\left(n, n^{\prime}\right) \leq x / k
$$

Now suppose $n=r w$ and $n^{\prime}=r w^{\prime}$ are two integers in $H_{j}^{(2)}$ with the same $r$. Then by (12), we have

$$
\left(w, w^{\prime}\right) \leq \frac{x}{k r}
$$

If both $w$ and $w^{\prime}$ have the same smallest prime factor $p_{j}$, and $p_{j}^{\alpha_{j}}\left\|w, p_{j}^{\alpha_{j}^{\prime}}\right\| w^{\prime}$ say, with $1 \leq \alpha_{j} \leq \alpha_{j}^{\prime}$, then it follows from (13) that $p_{j}^{\alpha_{j}} \leq \frac{x}{k r}$. At the same time, we also have the condition (on $n$ ) in $H_{j}^{(2)}$ that

$$
r p_{j}^{\alpha_{j}+1}>\frac{x}{k}
$$

This means that $\alpha_{j}$ is uniquely determined by the inequalities

$$
p_{j}^{\alpha_{j}} \leq \frac{x}{k r}, \quad p_{j}^{\alpha_{j}+1}>\frac{x}{k r} .
$$

We can now conclude that, for all $n \in H_{j}^{(2)}, n=r w$ with a given $r$, either

(i) $w=p_{j}^{\beta} \ldots$ for one value of $\beta$, which is determined uniquely by $p_{j}$; or

(ii) $w=p_{j}^{\beta} \cdots$, where $\beta$ takes exactly two values, one of which is $\alpha_{j}$ in (14) and another one larger than this. Both of these two values are determined uniquely by $p_{j}$. 
We now return to the sum

$$
\sum_{\substack{y<n \leq x+y \\ n \equiv a(\bmod k) \\ n \in H_{j}^{(2)}}} 1
$$

Those $n$ in case (i) contribute

$$
\sum_{1} \leq \sum_{r} \sum_{p_{j}} \sum_{\substack{\left.y_{\left(r p_{j}\right.}^{\alpha_{j}}\right)^{-1}<m \leq(x+y)\left(r p_{j} \alpha_{j}\right)^{-1} \\ m \equiv r p_{j} \alpha_{j} \\(\bmod k)}} 1
$$

Note that $\alpha_{j}$ in the inner summation is uniquely determined by $r$ and $p_{j}$. The innermost sum is clearly

$$
\leq x\left(k r p_{j}^{\alpha_{j}}\right)^{-1}+1
$$

Hence

$$
\begin{aligned}
\sum_{1} & \leq \frac{x}{k} \sum_{r} \sum_{p_{j}} \frac{1}{r p_{j}^{\alpha_{j}}}+\sum_{r} \sum_{p_{j}} 1 \\
& \leq \frac{x}{k} Q_{j}\left(\frac{x}{k}\right)+\sum_{r} \sum_{p \leq \xi / r} 1
\end{aligned}
$$

in view of the conditions in $H_{j}^{(2)}$ and the definition of $Q_{j}$. Hence, by the prime number theorem, we have

$$
\begin{aligned}
\sum_{1} & \leq \frac{x}{k} Q_{j}\left(\frac{x}{k}\right)+\frac{x}{k} \sum_{r} \frac{2}{r \log \frac{x}{k r}} \\
& \leq \frac{x}{k} Q_{j}\left(\frac{x}{k}\right)+2 \frac{x}{k} P_{j-1}\left(\frac{x}{k}\right) \\
& \leq \frac{K x(\log \log (x / k)+K)^{j-1}}{k \log (x / k)(j-1) !}
\end{aligned}
$$

The contribution of those $n$ in case (ii) above is

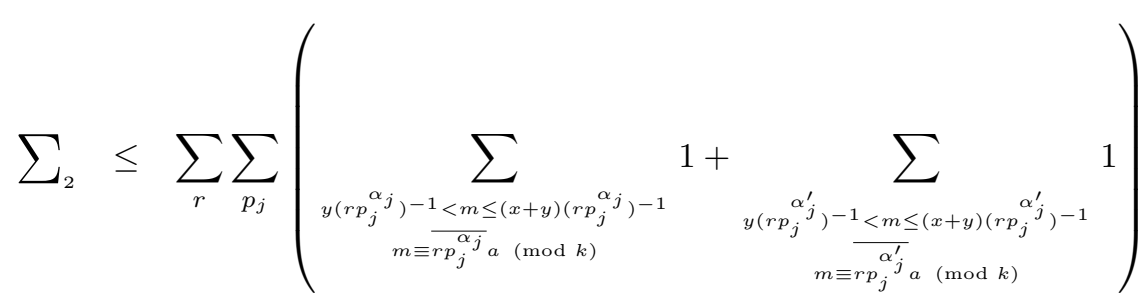

$$
\begin{aligned}
& \leq \sum_{r} \sum_{p_{j}}\left(\frac{x}{r p_{j}^{\alpha_{j}} k}+\frac{x}{r p_{j}^{\alpha_{j}^{\prime}} k}+2\right) \\
& \leq 4 \sum_{r} \sum_{p_{j}} \frac{x}{k r p_{j}^{\alpha_{j}}} \leq K \frac{x}{k} Q_{j}\left(\frac{x}{k}\right),
\end{aligned}
$$


as in the case of $\sum_{1}$. The case that $w$ has just one prime power can be verified separately by using Lemma 6 . Combining this with (15), we prove Lemma 8.

\section{Proof of the Theorem}

Again, when $k>x / 2$, the result is trivial. Therefore we may assume $k \leq x / 2$. Let $s \geq 1$ be given and set $\xi=x / k(>2)$. By the definition of $H_{j}(\xi)$, it is easy to see that if $n=p_{1}^{\alpha_{1}} p_{2}^{\alpha_{2}} \cdots p_{j}^{\alpha_{j}}$ does not belong to $H_{1} \cup H_{2} \cup \cdots \cup H_{j}$, then $p_{1}^{\alpha_{1}} p_{2}^{\alpha_{2}} \cdots p_{j-1}^{\alpha_{j-1}} p_{j}^{\alpha_{j}+1} \leq \xi$; and hence $n \leq \xi$. Thus

$$
\sum_{\substack{y<n \leq x+y \\ n \equiv a(\bmod ) \\ \omega(n) \leq s}} 1 \leq \sum_{\substack{y<n \leq x+y \\ n=a(\bmod k) \\ n \in H_{1} \cup \cdots \cup H_{s}}} 1+\sum_{\substack{n \leq \xi \\ \omega(n) \leq s}} 1 .
$$

Applying Lemma 8 to the first sum and Hardy-Ramanujan's inequality (3) to the second sum, we get the bound

$$
\frac{K x}{\varphi(k) \log (x / k)} \sum_{l=0}^{s-1} \frac{(\log \log (x / k)+K)^{l}}{l !}
$$

for the above right hand side. This proves (6) in our Theorem 1.

For $s \leq \log \log (x / k)$, we let $\lambda=s / L \leq 1$ where $L=\log \log (x / k)+K$. If $\lambda<1$, then the summation at the right hand side of (6) is

$$
\begin{aligned}
\sum_{l=0}^{s-1} \frac{1}{l !}(\log \log (x / k)+K)^{l} & =\frac{(\log \log (x / k)+K)^{s-1}}{(s-1) !} \sum_{l=0}^{s-1} \frac{(s-1)(s-2) \cdots(l+1)}{(\log \log (x / k)+K)^{s-l-1}} \\
& \leq \frac{(\log \log (x / k)+K)^{s-1}}{(s-1) !} \sum_{l=0}^{s-1} \lambda^{s-l-1} \\
& \leq \frac{(\log \log (x / k)+K)^{s-1}}{(s-1) !} \sum_{l=0}^{\infty} \lambda^{l} \\
& =\frac{(1-\lambda)^{-1}(\log \log (x / k)+K)^{s-1}}{(s-1) !}
\end{aligned}
$$

and if $\lambda \leq 1$, then the summation at the right hand side of (6) is

$$
\begin{aligned}
\sum_{l=0}^{s-1} \frac{1}{l !}(\log \log (x / k)+K)^{l} & =\frac{(\log \log (x / k)+K)^{s-1}}{(s-1) !} \sum_{l=0}^{s-1} \frac{(s-1)(s-2) \cdots(l+1)}{(\log \log (x / k)+K)^{s-l-1}} \\
& =\frac{(\log \log (x / k)+K)^{s-1}}{(s-1) !} \sum_{l=0}^{s-1}\left(1-\frac{1}{L}\right)\left(1-\frac{2}{L}\right) \cdots\left(1-\frac{s-l-1}{L}\right) \\
& \leq \frac{(\log \log (x / k)+K)^{s-1}}{(s-1) !} \sum_{l=0}^{s-1} e^{-\frac{1}{L}} e^{-\frac{2}{L}} \cdots e^{-\frac{s-l-1}{L}} .
\end{aligned}
$$


The last summation over $l$ is

$$
\begin{aligned}
& =\sum_{l=0}^{s-1} e^{-l(l+1) /(2 L)} \leq \sum_{0 \leq l \leq \sqrt{L}} 1+\sum_{\sqrt{L}<l \leq s-1} e^{-l^{2} /(2 L)} \\
& \ll \sqrt{L}+\int_{\sqrt{L}}^{\infty} e^{-t^{2} /(2 L)} d t \ll \sqrt{L}+\sqrt{L} \int_{1}^{\infty} e^{-t^{2}} d t \ll \sqrt{L} .
\end{aligned}
$$

This completes the proof of our Theorem 1.

Acknowledgment. The authors wish to express their gratitude to the referee for the thoughtful and gracious comments and suggestions.

\section{REFERENCES}

[1] H. Davenport, Multiplicative Number Theory, 3rd Edition, Graduate Texts in Math. 74 Berlin-Heidelberg-New York 2000.

[2] H. Halberstam and H.E. Richert, Sieve Method, Academic Press 1974, London.

[3] G. H. Hardy and S. Ramanujan, The normal number of prime factors of a number $n$, Quarterly J. Math. 48 (1917), 76-92.

[4] A. Hildebrand and G. Tenenbaum, On the number of prime factors of an integer, Duke Math. J. 56, No. 3, 1988, 471-501.

[5] E. Landau, Handbuch der Lehre von der Verteilung der Primzahlen, Vol. 1. Leipzig, 1909.

[6] H. L. Montgomery and R. C. Vaughan, Multiplicative Number Theory I. Classical Theory, Cambridge Studies in Advanced Mathematics 97, Cambridge University Press 2007, New York.

[7] M. Orazov, Analogue of the Brun-Titchmarsh inequality. (Russian) Izv. Akad. Nauk Turkmen. SSR Ser. Fiz.-Tekhn. Khim. Geol. Nauk (1982), no. 2, 90-91.

[8] L. G. Sathe, On a problem of Hardy and Ramanujan on the distribution of integers having a given number of prime factors, J. Indian Math Soc. 17 (1953), 63-141; 18 (1954), 27-81.

[9] A. Selberg, Note on a paper by L. G. Sathe, J. Indian Math Soc. 18 (1954), 83-87.

[10] C. A. Spiro, Extensions of some formulae of A. Selberg, Internat. J. Math. Math. Sci. 8 (1985), no. 2, 283-302.

[11] C. Tudesq, Étude de la loi locale de $\omega(n)$ dans de petits intervalles. (French. English summary) [Study of the local law of $\omega(n)$ in small intervals] Ramanujan J. 4 (2000), no. 3, 277-290.

[12] R. Warlimont and D. Wolke, Über quadratfreie Zahlen mit vorgeschriebener Primteileranzahl. (German) Math. Z. 155 (1977), no. 1, 79-82. 
Department of Mathematical Sciences, University of Memphis, Memphis, TN 38152, U.S.A.

E-mail address: tchan@memphis.edu

Department of Mathematics, Simon Fraser University, Burnaby, B.C., Canada V5A 1S6

E-mail address: kkchoi@math.sfu.ca

Department of Mathematics, The University of Hong Kong, Hong Kong, SAR, China

E-mail address: kmtsang@maths.hku.hk 